\title{
Iterative Orientation Tuning in V1: A Simple Cell Circuit with Cross-Orientation Suppression
}

\author{
Marina Kolesnik $^{1}$ and Alexander Barlit \\ Fraunhofer Institute for Media Communication, Schloss Birlinghoven, D-53754 \\ Sankt-Augustin, Germany
}

\begin{abstract}
An iterative model for contrast detection, which accounts for the contrast invariance of orientation preference, has been recently suggested [16]. The work here extends the iterative model by incorporating a cross-oriented suppression of simple cells in the primary visual cortex (V1). The modified model has a better performance in terms of robustness to noise, generates sharper edge responses while suppressing weak edges, and converges faster on equilibrium. The model exhibits a higher level of contrast invariance of orientation preference generating a clear pattern of edges in natural images.
\end{abstract}

\section{Introduction}

The orientation selectivity of simple cells in V1, as proposed by Hubel and Wiesel [1], derives from an oriented arrangement of input from the lateral geniculate nucleus (LGN): ON-centre LGN inputs have receptive fields (RFs) centres aligned over simple cell's ON subregions, and similarly for OFF-centre inputs. Because of this input arrangement, simple cells perform a linear spatial summation of light intensity in their fields and have an elongated shape of their RFs. A traditional linear model performs spatial summation of input signals from the LGN followed by a rectification [2], [3]. Although many aspects of simple cell responses are consistent with this linear model, there also are important violations of linearity. For example, scaling the contrast of a stimulus would identically scale the responses of a linear cell. At high contrasts, however, the responses of simple cells show clear saturation. Such behaviour of the simple cells is referred to as contrast invariance of orientation selectivity [4].

Several biophysical models have attempted to address the nonlinearities of simple cells by extending the linear model to include a gain control stage [5], [6], [7]. It is suggested the response of a simple cell is governed by shunting inhibition - the divisive normalization of the cell activity due interaction with other cells. The shunting inhibition controls the gain of the transformation of the cell's input current to output membrane potential [8]. A followed rectification stage converts the latter into a firing rate of the cell. These biophysical models laid the foundation for the development of computational models for contrast detection in images [10], [11], [12]. A typical architecture of such a model is built upon a simple cell circuit, which is composed of segregated ON- and OFF-data streams interacting via mechanism of opponent inhibition suggested by Ferster [9].

None of these models, however, explicitly employs the impressive regularity in a spatial layout of simple cells. Back in 1974, Hubel and Wiesel [14], [15] discovered that when

\footnotetext{
${ }^{1}$ To whom correspondence should be addressed: marina.kolesnik@,imk.fraunhofer.de
} 
orientation preference of cells in the ocular dominance stripes of V1 is related to their position, an astonishingly systematic organisation emerges: the orientation preference changes linearly with position across V1. For evolution was likely to select developmental rules that produce orientation preference maps which are sufficiently optimised in terms of length of neuronal connections, local interaction of spatially close simple cells ought to have an important role in their functionality. A first attempt to utilize the regularity of orientation preference layout for the detection of luminance changes in images has been made in [16]. The model achieves a significant level of contrast invariance of orientation selectivity due to the iterative processing of visual input and local amplification of responses of simple cells.

Another aspect of the behaviour of simple cells, which was not accounted for in [16], lies in the fact that simple cells are subject to cross-oriented inhibition; the responses to an optimally oriented stimulus can be diminished by superimposing an orthogonal stimulus that is ineffective in driving the cell when presented alone [17], [18]. This work specifically addresses this aspect of the behaviour of simple cells. The model here extends the iterative model in [16] by incorporating a mechanism of orthogonal suppression of spatially close simple cells. The processing of natural images demonstrates a higher level of contrast invariance with respect to isolated luminance changes in images and generates a clearer pattern of edges.

\section{Model neural network}

A neural circuit of the model (Fig. 1) consists of two ON- and OFF-pathways interacting via a mechanism of opponent inhibition. Video input is processed sequentially by retinaLGN cells followed by a simple cell circuit in V1. A key feature of the model is the iterative processing of video input, which imitates an instance when the eye is fixating a particular object and the processing of still visual input might undergo several iterative cycles. Due to local intracortical interaction, neural responses of simple cells to constant visual input would vary at different iterations. The local interaction of simple cells is governed by morphology of their layout. We adopt an Icecube model to describe the spatial layout of simple cells. Due to the process of local interaction, the activation of a simple cell causes excitation of neighbouring cells which are close within the Icecube layout.

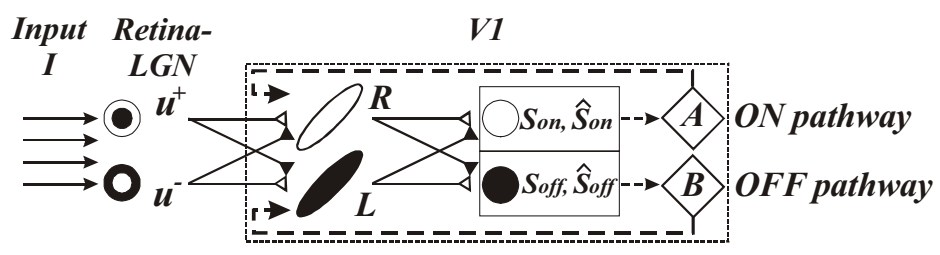

Figure 1. The architecture of the model neural network. Simple cells of V1 are engaged in the crosschannel inhibition, cross-oriented suppression and local amplification. Triangles at the end of lines denote the excitatory input; filled-in-black triangles denote the inhibitory input. Two dashed lines show the local amplification of simple cell subfields due to the instantaneous activation of simple cells.

The first processing stage consists of retinal ganglion cells with centre-surround 
receptive fields (RFs) [19]. The retinal ON and OFF cells are modelled at each spatial position by the difference of video input and its convolution with a 2-dimensional Gaussian kernel (A1), [20]. Retinal ON and OFF ganglion cells synapse mainly onto respective ON and OFF cells of the LGN. In the model, retinal inputs do not change while passing the LGN.

Simple cells in V1 are driven by oriented input from the LGN. Physiological studies on simple cell responses recorded in cat striate cortex suggest that elongated sensitivity profile of a simple cell subfield is best modelled by a difference of two elongated Gaussians (A2, A3). Each ON cell subfield receives excitatory input from the LGN ON cells beneath it and is inhibited by LGN OFF cells at the same retinotopic position (A3).

In addition, simple cells undergo local interaction, which amplifies the activity of cells of same pathway. Each simple ON cell undergoes additive amplification received from those simple ON cells that are spatially close in Icecube layout; the same ON cell is inhibited by proximate simple OFF cells (A3). The reverse arrangement holds true for simple OFF cells. Final activation of a simple cell results from the cross-channel inhibition of the ON and OFF simple cell subfields (A4).

\subsection{Intracortical excitation and inhibition of simple cells}

All simple cells are considered to be stacked into a 3-D array (Fig. 2, left), in which two coordinates define the spatial (retinotopic) position of the cell and the remaining third coordinate is related to the cell's preferred orientation. Optical imaging studies on patterns of activation across a region of monkey cortex [21] revealed iso-orientation contours connecting the simple cells of neighbouring orientation columns via points of singularity. (Fig. 2, right). We suggest that this systematic wiring is employed in the local intracortical interaction of simple cells.

Two types of local interaction are employed in the model. First, the iso-oriented contours are used for the propagation of inhibitory signals to cells at orthogonal orientation. The activity of each simple cell is inhibited by four neighbouring cells of the same spatial layer (Fig.2) but tuned to orthogonal orientation (A5). This mechanism of cross-orientation suppression affects the activity of simple cells in two ways. On one hand, the response of a simple cell is cancelled out by the activity of retinotopically close cells of orthogonal orientation if these are strongly activated. On the other hand, the response of a strongly activated cell will be only weakly suppressed if retinotopically close cells of orthogonal orientation are themselves weakly activated. This orthogonal suppression eliminates weak spurious responses while sharpening strong responses of simple cells.

Second type of local interaction is the amplification of proximate cells of the same pathway. This local amplification enhances responses of both retinotopically proximate cells and cells at similar orientations. The strength of amplification is an inverse function of squared distance (A6) and the excitation of proximate cells effectively decays within the distance of 3 units. Due to the weighting factor $\omega=16$ in (A6), the effect of amplification affects only one neighbouring cell in all 8 orientations within the spatial layer, and about 6 neighbouring cells in the orientation column ( 3 orientations both up and down the column). Consequently, the local amplification triggers a process of selective orientation tuning enhancing responses of cells of "proper" orientations at retinotopically close positions, whereas the cross-orientation inhibition serves as a mechanism of selective suppression, which affects locally the activity of all cells that receive conflicting visual input with no 
distinguished orientation.

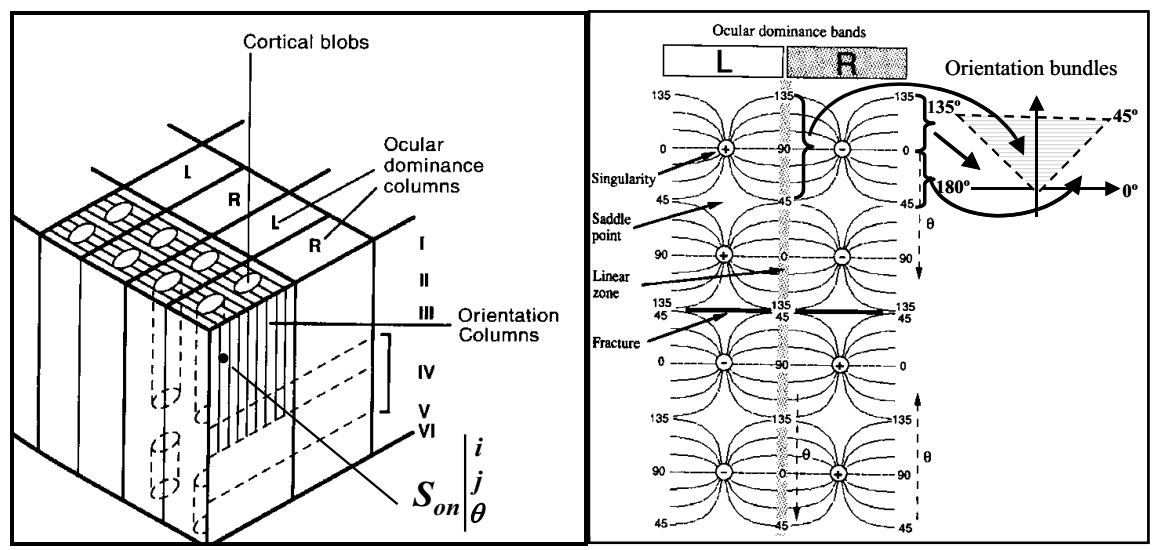

Figure 2. A schematic diagram of the spatial layout of V1 - the Icecube layout. Left: the spatial layout of simple cells is modelled by the 3-D array consisting of spatial layers and orientation columns. Each element of the array, $(i, j, \theta$,$) has two spatial coordinates, i$ and $j$, for position within the layer and one orientation coordinate, $\theta$, for position in the orientation column. It is assumed that each 3-D position contains a pair of ON and OFF cells, $S_{\text {on }}$ and $S_{\text {off }}$ This array layout is repeated twice for both contrast polarities $p=1,2$. Right: Schematic presentation of relationship between ocular dominance bands and the organisation of orientation selectivity in the visual cortex (Obermayer and Blasdel [21]). Cells along "iso-oriented contour" have the same optimal orientation. Orientations in each bundle of iso-contours range within $90^{\circ}$. Adjacent contours represent orientations $11.25^{\circ}$ apart. A complete circle around each singularity represents a rotation from $0^{\circ}$ to $180^{\circ}$. Adjacent iso-contour bundles linked at points of singularity range within two complementary sectors of $90^{\circ}$ each (shown in black arrows).

\subsection{The iterative processing of visual input}

The local intracortical interaction alters the response of simple cells to constant visual input when the model proceeds to the next iteration and the processing cycle is repeated. Due to the local amplification, responses of simple cells in the vicinity of pronounced luminance changes would increase at later iterations. Two mechanisms help the model to reach equilibrium: 1) cross-channel inhibition (A4) does not let the activity of simple cells to grow indefinitely; 2) cross-orientation suppression (A5) limit the propagation of excitation onto cells which do not receive salient oriented excitation from visual input. Typically, it takes 4-5 iterations until the growth of simple cell activation slows down to $3 \%-4 \%$ per iterative cycle at which point the processing is interrupted.

The iterative processing combined with the cross-channel inhibition leads to a key property of the model - the contrast invariance of orientation preference: because of the divisive non-linear normalisation embodied in the denominator of (A4), the cross-channel inhibition tends to boost weaker responses over the stronger ones [16], so that at subsequent iterations the response to low-contrast luminance changes grows faster than the response to contrastive luminance changes. 


\section{Processing results and conclusions}

In this work we have proposed the extension of the iterative model for contrast detection [16], which is based on the mechanism of orientation selectivity in the primary visual cortex. The extended model explicitly incorporates the mechanism of cross-orientation suppression. An example of edge image processed by the extended model is shown in Fig. 3. Comparison of processing results generated by the extended model against earlier ones reported in [16] shows that the incorporation of the mechanism of cross-orientation suppression increases model's robustness to noise; generates sharper edge responses while suppressing weak edge responses; accelerates the convergence of the model on equilibrium and exhibits a higher level of contrast invariance with respect to contrast variations in visual images.

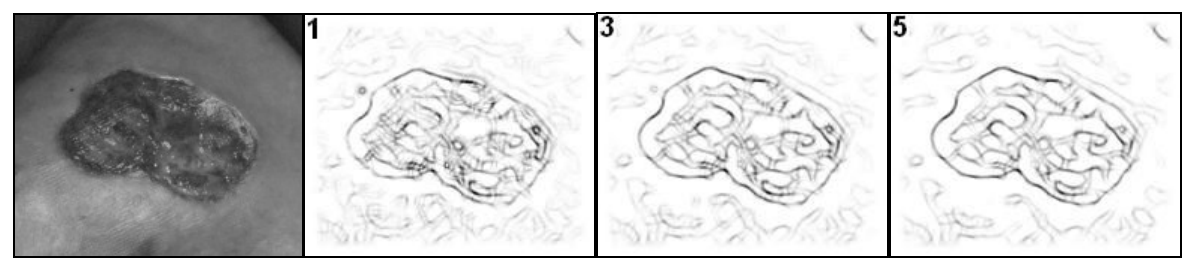

Figure 3. Input image of a wound and edge images after $1^{\text {st }}, 3^{\text {rd }}$, and $5^{\text {th }}$ iteration. All result images are obtained by rectifying the sum of activities of ON and OFF subfields, $R$ and $L$, minus the absolute value of their difference and by pooling together the results of both contrast polarities for all twelve orientations. Weak edges disappear and strong edges are amplified as the model proceeds through iterative cycles. This process diminishes spurious responses both for skin and the wound region.

\section{References}

1. Hubel, D., H., Wiesel, T., N.: Receptive fields, binocular interaction and functional architecture in the cat's visual cortex. Journal of Psychology, 160:106-154, 1962.

2. Movshon, J., A., Thompson, I., D., Tolhurst, D., J. Spatial summation in the receptive fields of simple cells in the cat's striate cortex. Journal of Physiology (London), 283:53-77, 1978.

3. Carandini, M., Ferster, D. A tonic hyperpolarization underlying contrast adaptation in the cat visual cortex. Science 276:949-952. 1997.

4. Sclar, G., Freeman, R.: Orientation selectivity in the cat's striate cortex is invariant with stimulus contrast. Experimental Brain Research, 46, (1982) 457-461.

5. Albrecht, D., G., Geisler, W., S. Motion sensitivity and the contrast response function of simple cells in the visual cortex. Visual Neuroscience. 7:531-546.

6. Heeger, D., J. Nonlinear model of neural responses in cat visual cortex. In M. Landy, J. A. Movshon (eds.): Computational models of visual processing. Cambridge, MIT: 119-133, 1991.

7. DeAngelis G., C., Robson, J., G., Ohzawa, I., Freeman, R., D. The organization of suppression in receptive fields of neurons in the cat's visual cortex. J. Neurophysiology. 68:144-163. 1992.

8. Carandini, M., Heeger, D., J. Summation and division by neurons in visual cortex. Science, 264:1333-1336, 1994.

9. Ferster, D.: The synaptic inputs to simple cells in the cat visual cortex. In: D. Lam and G. Gilbert (eds.): Neural mechanisms of visual perception, Ch. 3, Portfolio Publ. Co, The Woodlands, Texas: 63-85, 1989.

10. Pessoa, L., Mingolla, E., Neumann, H.: A contrast- and luminance-driven multiscale network 
model of brightness perception. Vision Research, 35:2201-2223, 1995.

11. Neumann, H., Pessoa, L., Hansen, Th.: Interaction of ON and OFF pathways for visual contrast measurement. Biological Cybernetics, 81:515-532, 1999.

12. Hansen, Th., Neumann, H. A model of V1 visual contrast processing utilizing long-range connections and recurrent interactions. In Proc. of the International Conference on Artificial Neural Networks, Edinburgh, UK, Sept. 7-10:61-66, 1999.

13. Hansen, Th., Baratoff, G., Neumann, H.: A simple cell model with dominating opponent inhibition for robust contrast detection. Kognitionswissenschaft, 9:93-100, 2000.

14. Hubel, D., H., Wiesel, T., N.: Sequence regularity and geometry of orientation columns in the monkey striate cortex. Journal of Comparative Neurology, 158:267-294, 1974.

15. Hubel, D., H., Wiesel, T., N.: Functional architecture of macaque monkey visual cortex. Proceedings of the Royal Cosiety of London, B, 198:1-59, 1977.

16. Kolesnik, M., Barlit, A., Zubkov, E. Iterative Tuning of Simple Cells for Contrast Invariant Edge Enhancement. Proc. of the $2^{\text {nd }}$ International Workshop on Biologically Motivated Computer Vision (BMCV'2002), 27-37, 2002.

17. Morrone, M., C., Burr, D., C., Maffei, L. Functional implications of cross-orientation inhibition of cortical visual cell. 1. Neurophysiological evidence. Proc. Royal Society London [Biol.], 216:335-354, 1982.

18. Bonds, A., B. Role of inhibition in the specification of orientation selectivity of cells in the cat striate cortex. Visual Neuroscience, 2:41-55.

19. Kuffler, S., W.: Discharge patterns and functional organization of mammalian retina. Journal of Neurophysiology, 16:37-68, 1953.

20. Grossberg, S., Raizada, R., D., S.: Contrast-sensitive perceptual grouping and object-based attention in the laminar circuits of primary visual cortex. CAS/CNS TR-99-008, Boston University: 1-35, 1999.

21. Obermayer, K., Blasdel, G., G. Geometry of orientation and ocular dominance columns in monkey striate cortex. Journal of Neuroscience. 13:4114-4129. 1993.

\section{Appendix: Model equations}

Responses of retinal ganglion ON and OFF cells, $u_{i j}^{+}$and $u_{i j}^{-}$, are given by:

$$
\begin{aligned}
& X_{i j}=I_{i j}-G_{\sigma} * I_{i j} \\
& u_{i j}^{+}=\left[X_{i j}\right]^{+}, \quad u_{i j}^{-}=\left[-X_{i j}\right]^{+}
\end{aligned}
$$

where $*$ is the spatial convolution operator and $G_{\sigma}$ is a centre Gaussian with standard deviation $\sigma=3$ sampled within a filter mask of $17 \times 17$ pixels. Video input $I$ is normalised to $[0,1]$.

Simple cells are modelled for twelve discrete orientations $\theta=0^{\circ}, 15^{\circ}, \ldots, 165^{\circ}$, and two opposite contrast polarities $p=1,2$ :

$$
\begin{aligned}
& D_{\theta \sigma_{M} \sigma_{m} \tau}=G_{\theta \sigma_{M} \sigma_{m} \tau}-G_{\theta \sigma_{M} \sigma_{m}-\boldsymbol{\tau}} \\
& G_{\theta \sigma_{M} \sigma_{m} \tau}=\frac{1}{2 \pi} \exp \left[-\frac{1}{2}\left((\mathbf{x}-\boldsymbol{\tau})^{T} \mathbf{R}^{T} \mathbf{C R}(\mathbf{x}-\boldsymbol{\tau})\right)\right] \\
& \mathbf{C}=\left(\begin{array}{cc}
1 / \sigma_{m}^{2} & 0 \\
0 & 1 / \sigma_{M}^{2}
\end{array}\right), \quad \mathbf{R}=\left(\begin{array}{cc}
\cos \theta & \sin \theta \\
-\sin \theta & \cos \theta
\end{array}\right)
\end{aligned}
$$

where $\mathbf{x}^{T}=(i, j)$ denotes the position $(i, j), \boldsymbol{\tau}^{T}=(\cos \theta, \sin \theta)$ is relative offset for two Gaussian 
lobes from their central position $(i, j)$, and the space constants $\sigma_{m}=1$ and $\sigma_{M}=4$ define the degree of filter's elongation.

At each position, $(i, j)$, and for each orientation, $\theta$, and polarity, $p$, the model has a simple cell with two parallel elongated parts: an ON subfield, $R_{i, j, \theta, p}$, which receives excitation from LGN ON cells beneath it and is inhibited by LGN OFF cells at the same position; and an OFF subfield, $L_{i, j, \theta, p}$, for which the reverse relation with regard to the LGN channels holds true. In addition, each ON subfield, $R_{i, j, \theta, p}$, receives excitatory input, $A_{i, j, \theta, p}$, from all simple ON cells that are spatially close to position $(i, j)$ in the Icecube layout, and is inhibited by input $B_{i, j, \theta, p}$ from all close OFF cells. The reverse arrangement holds true for the computation of the activation level of each OFF subfield, $L_{i, j, \theta, p}$. The mutual amplification-inhibition of neighbouring cells is a time varying function which is updated iteratively:

$$
\begin{aligned}
& R_{i, j, \theta, p}^{n}=\left[\left(u_{i j}^{+}+A_{i, j, \theta, p}^{n}-u_{i j}^{-}-B_{i, j, \theta, p}^{n}\right) *\left[D_{\theta \sigma_{M} \sigma_{m} \tau}^{p}\right]^{+}\right]^{+} \\
& L_{i, j, \theta, p}^{n}=\left[\left(u_{i j}^{-}+B_{i, j, \theta, p}^{n}-u_{i j}^{+}-A_{i, j, \theta, p}^{n}\right) *\left[-D_{\theta \sigma_{M} \sigma_{m} \tau}^{p}\right]^{+}\right]^{+}
\end{aligned}
$$

where $n$ is the iteration number and the values of $A_{\theta, p}, B_{\theta, p}$ at initial iteration are set to zero.

The activation of a simple ON cell at iteration $n, S_{o n}$, is obtained as the steady-state solution of inhibitory shunting interaction:

$$
S_{o n}^{n}=\left[\left(R^{n}-L^{n}\right) /\left(1+R^{n}+L^{n}\right)\right]^{+}
$$

Here variables occur for all positions, orientations and polarities; indexes $i, j, \theta$, and $p$ are omitted to simplify notations. Activation of a simple OFF cell is obtained by interchanging $R^{n}$ and $L^{n}$.

Simple cells are engaged in the cross-orientation inhibition, so that the cell's activity at position $(i, j, \theta)$, is inhibited by four neighbouring cells within the spatial layer (see caption to Fig.2) that are tuned to the orthogonal direction:

$$
\begin{aligned}
& \hat{S}_{i, j, \theta}^{n}=\left[S_{l, m, \vartheta}^{n}-\left(S_{l+1, m, \vartheta}^{n}+S_{l-1, m, \vartheta}^{n}+S_{l, m+1, \vartheta}^{n}+S_{l, m-1, \vartheta}^{n}\right) / 4\right]^{+}, \\
& \left\{\begin{array}{l}
\vartheta=\theta+\pi / 2 \text { if } 0 \leq \theta<\pi / 2 \\
\vartheta=\theta-\pi / 2 \text { if } \pi / 2 \leq \theta<\pi
\end{array}\right.
\end{aligned}
$$

Excitatory input, $A_{i, j, \theta}^{n}$, from neighbouring cells, is an inverse function of squared distance:

$$
\begin{aligned}
& A_{i, j, \theta}^{n}=\mu \sum_{l, m, \vartheta} \frac{\hat{S}_{(o n), l, m, \vartheta}^{n}}{D^{2}[(l, m, \vartheta),(i, j, \theta)]}, \\
& D^{2}[(l, m, k),(i, j, \theta)]=\omega\left((l-i)^{2}+(m-j)^{2}\right)+(\theta-\vartheta)^{2}
\end{aligned}
$$

here $\mu$ - is a scaling factor set to: $\mu=0.1$, and $\omega-$ is a weighting factor set to: $\omega=16$. The computations above are repeated twice for both polarities. Excitatory input $B$ to an OFF cell is obtained similarly by substituting $S_{\text {off }}$ into (A6). 